\title{
Effect of Lime, Phosphorus and Boron on Yield and Chemical Composition of Maize (Zea mays L.) and Properties of Acid Soil of Nagaland
}

\author{
Merasenla Ao and Y. K. Sharma* \\ Department of Agricultural Chemistry and Soil Science, SASRD, Nagaland University, \\ Medziphema, Nagaland, India \\ *Corresponding author
}

\begin{tabular}{|l|}
\hline K e y w o r d s \\
Maize, Lime, \\
Phosphorus, Boron, \\
Nutrient uptake, \\
Soil properties
\end{tabular}

\section{A B S T R A C T}

A pot experiment was conducted during kharif season of 2016-17 and 2017-18 in completely randomized design with two lime levels ( 0 and $1 / 4$ lime of LR), four phosphorus levels $\left(0,13.4,26.8\right.$ and $\left.40.2 \mathrm{mg} \mathrm{P}_{2} \mathrm{O}_{5} \mathrm{~kg}^{-1}\right)$ and three boron levels $(0,0.45$ and $0.90 \mathrm{mg} \mathrm{B}$ $\mathrm{kg}^{-1}$ ) to study the treatment effect on nutrient content and uptake of maize and soil properties. It was observed that lime had a significant effect on biomass yield, nutrient content and uptake of maize. Application of $1 / 4$ lime of LR increased the grain yield by $25.3 \%$ and $24.0 \%$ and stover yield by $24.1 \%$ and $22.0 \%$ during first and second year, respectively over control. Effect of phosphorus was significant on the yield, nutrient content and uptake of maize, where application of $26.8 \mathrm{mg} \mathrm{P}_{2} \mathrm{O}_{5} \mathrm{~kg}^{-1}$ proved optimum level. Conversely, $\mathrm{B}$ content in both grain and stover remained unaffected by $\mathrm{P}$ application. Application of $26.8 \mathrm{mg} \mathrm{P}_{2} \mathrm{O}_{5} \mathrm{~kg}^{-1}$ increased grain yield by $23.6 \%$ and $23.0 \%$ and stover yield by $19.7 \%$ and $20.0 \%$ during first and second year, respectively over control. Application of boron had a significant effect on yield where highest grain and stover yield was recorded at $0.90 \mathrm{mg} \mathrm{B} \mathrm{kg}^{-1}$ which increased grain yield by $8.7 \%$ and $13.1 \%$ and stover yield by $9.9 \%$ and $11.4 \%$ during first and second year, respectively. Significant effect of B on nutrient content was observed in N, P and B content in grain and stover, while significant effect of B on nutrient uptake was observed in $\mathrm{N}, \mathrm{P}, \mathrm{K}$ and $\mathrm{B}$ uptake in grain and stover. Among the soil properties, application of $1 / 4 \mathrm{lime}$ of LR significantly increased soil $\mathrm{pH}$, base saturation, available $\mathrm{N}, \mathrm{P}, \mathrm{K}, \mathrm{Ca}$ and $\mathrm{B}$, while it decreased exchangeable $\mathrm{H}^{+}, \mathrm{Al}^{3+}$ and total potential acidity of post-harvest soil. Significant effect of $\mathrm{P}$ was observed on available $\mathrm{P}$ and exchangeable $\mathrm{Ca}$ of post-harvest soil, while effect of boron was significant only on the available boron of post-harvest soil.

\section{Introduction}

Maize (Zea mays L.) globally recognized as the "Queen of cereals" belongs to the family of grasses (Poaceae) and is widely cultivated throughout the world. In India, maize is grown throughout the year and is predominantly a kharif crop with a total area of 9.18 million hectare (Agricultural Statistics at a Glance, 2019). Soils with good organic matter content having high water holding capacity with neutral $\mathrm{pH}$ are considered good for higher productivity. One of the major problems encountered by the farmers in maize 
producing areas in the North Eastern Regions of India is the soil acidity. Acid soils have high Aluminium (Al) content which leads to grain yield losses up to $60 \%$ (The et al., 2006). Soil acidity has a negative effect on crops mainly through $\mathrm{P}$ unavailability from $\mathrm{P}$ fixation in soils whereby the $\mathrm{Fe}$ and $\mathrm{Al}$ soil components (sesquioxides) fix sizeable quantities of $\mathrm{P}$. Therefore, in $\mathrm{P}$ fixing acid soils, combined lime and $\mathrm{P}$ application is necessary for increased availability of the applied $\mathrm{P}$ for plant uptake. Application of lime also eliminates actual and exchange acidities, minimizes hydrolytic acidity and raises the calcium content in the soil. Boron is one of the most deficient micronutrient in crops next to zinc on a global scale. Deficiency of boron is relatively common in acid, sandy soils and being highly mobile is easily leached (Nazir et al., 2016). In Nagaland due to acidity- induced soil fertility problems and traditionally minimal use of mineral fertilizers, there is a need for soil acidity management and nutrient management in the region. Maize is a heavy feeder which requires higher amounts of nutrients to maintain higher production. Use of inadequate quantity of fertilizers coupled with declining native/original soil fertility often leads to nutrient deficiencies and reduced production of this crop. Therefore, from this perspective, the investigation was carried out to study the effect of lime, phosphorus and boron on yield, nutrient content and their uptake in maize and properties of the acid soil of Nagaland.

\section{Materials and Methods}

A pot experiment was conducted in the Department of Agricultural Chemistry and Soil Science, SASRD, Nagaland University, Medziphema during the kharif season of 2016-2017 and 2017-2018 with maize (RCM75 ) as test crop. The experimental site lies at $25^{\circ} 45^{\prime} 15.95 " \mathrm{~N}$ latitude and $93^{\circ}$ 51' 44.71"
E longitude at an elevation of 310 meter above mean sea level. The average rainfall varies between 2000 and $2500 \mathrm{~mm}$. The experiment was conducted in earthen pots of $30 \mathrm{~cm}$ diameter, filled with $15 \mathrm{~kg}$ of soil. The experimental soil was sandy clay loam in texture with the initial soil properties as $\mathrm{pH}$ 5.2 , organic carbon $15.4 \mathrm{~g} \mathrm{~kg}^{-1}$, available $\mathrm{N}, \mathrm{P}$

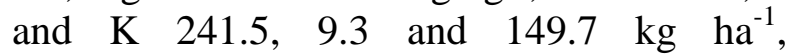
respectively, available $\mathrm{B} 0.52 \mathrm{mg} \mathrm{kg}^{-1}$ and lime requirement $6.6 \mathrm{t} \mathrm{ha}^{-1}$. Two lime levels ( 0 and $1 / 4$ lime of LR), four phosphorus levels (0, 13.4, 26.8 and $\left.40.2 \mathrm{mg} \mathrm{P}_{2} \mathrm{O}_{5} \mathrm{~kg}^{-1}\right)$ and three boron levels $(0,0.45$ and $0.90 \mathrm{mg} \mathrm{B}$ $\mathrm{kg}^{-1}$ ) were tested in completely randomized design with three replications. Lime, phosphorus and boron levels were developed through calcite $\left(\mathrm{CaCO}_{3}\right)$, single superphosphate and borax, respectively. Recommended dose of nitrogen and potassium (44.6 mg N kg ${ }^{-1}$ and $22.3 \mathrm{mg} \mathrm{K}_{2} \mathrm{O}$ $\mathrm{kg}^{-1}$ ) were supplied through urea and muriate of potash, respectively. Calculated amount of lime was applied 10 days before sowing. Half dose of $\mathrm{N}$ and full dose of phosphorus, potassium and boron were applied one day before sowing of maize. Remaining dose of nitrogen was applied in two splits i.e. half at 30 DAS and the remaining half at 60 DAS as top dressing. The soil was mixed properly after fertilizer and lime application. Three seeds in each pot were sown at optimum soil moisture to ensure germination on May 28, 2016 and May 25, 2017. Thinning was done 10 days after germination and one plant in each pot was allowed to grow. Weeding was done at regular interval and standard agronomic practices were adopted during the entire crop growing period. Data on grain and stover yield were recorded. Nitrogen content in plant samples was determined by Kjeldahl method, while phosphorus, potassium and calcium in plant samples were determined in diacid $\left(\mathrm{HNO}_{3}: \mathrm{HClO}_{4}\right.$ in the ratio of $\left.10: 4\right)$ extract by advocating standard procedure (Jackson, 1973). Phosphorous in the digested 
sample was determined by vanado-molybdate yellow colour method, $\mathrm{K}$ by flame photometer and $\mathrm{Ca}$ by versenate (EDTA) method. Boron content in plant samples was determined by dry ashing of the ground sample in a muffle furnace $\left(550^{\circ} \mathrm{C}\right.$ for $\left.1 \mathrm{~h}\right)$ and subsequent extraction with $0.36 \mathrm{~N} \mathrm{H}_{2} \mathrm{SO}_{4}$ (Gaines and Mitchell, 1979) and plant extract of $\mathrm{B}$ was determined by curcumin method as described by Dible et al., 1954. The nutrient uptake in grain and stover of maize was calculated by multiplying the nutrient content in grain and stover with their corresponding yield. The $\mathrm{pH}$, base saturation, organic carbon, available $\mathrm{K}$ and exchangeable $\mathrm{Ca}$ were analyzed using standard methods (Jackson, 1973). Available nitrogen was determined by alkaline permanganate method (Subbiah and Asija, 1956). For available $P$, soil samples were extracted with Bray P-1 extractant (Bray and Kurtz, 1945) and phosphorus content in soil extract was determined as described by Jackson (1973). The available boron in soil was determined by curcumin method as described by Dible et al., (1954). Exchangeable $\mathrm{Al}^{3+} \& \mathrm{H}^{+}$and total potential acidity were determined using standard methods described by Baruah and Barthakur (1997). The exchangeable $\mathrm{H}^{+}$was estimated by the difference between exchangeable acidity and exchangeable $\mathrm{Al}^{3+}$ (Sarma et al., 1987). The data were analyzed statistically to compare the treatment effects (Panse and Sukhatme, 1961).

\section{Results and Discussion}

\section{Biomass yield}

Effect of lime, phosphorus as well as boron was observed to be positively significant with respect to grain and stover yield (Table 1). Both grain and stover yield was found to be highest in the treatment where lime was applied i.e., at $1 / 4$ lime of LR during first and second year. Application of $1 / 4$ lime of LR increased grain yield by $25.3 \%$ and $24.0 \%$ and stover yield by $24.1 \%$ and $22.0 \%$ during first and second year, respectively over control. Liming is an important practice to achieve optimum yields of all crops grown on acid soils because it increases $\mathrm{pH}$ and reduces acidity-related constraints (Fageria and Baligar, 2008). Furthermore, lime application enhanced yield attributes which resulted in increased grain yield. The maximum grain and stover yield was recorded at $40.2 \mathrm{mg}$ $\mathrm{P}_{2} \mathrm{O}_{5} \mathrm{~kg}^{-1}$, which was found to be at par with the application of $26.8 \mathrm{mg}_{2} \mathrm{O}_{5} \mathrm{~kg}^{-1}$ for both grain and stover during both years. It was observed that application of $26.8 \mathrm{mg}_{2} \mathrm{O}_{5} \mathrm{~kg}^{-1}$ increased grain yield to the extent of $23.6 \%$ and $23.0 \%$ and stover yield $19.7 \%$ and $20.0 \%$ during first and second year, respectively over control. The increase in biomass yield due to phosphorus application may be due to enhancement in growth and yield attributes. Similar results have also been reported by Kumar et al., (2017); Ngosai et al., (2018). Significantly highest biomass yield was recorded at $0.90 \mathrm{mg} \mathrm{B} \mathrm{kg}^{-1}$ during first and second year. It was also observed that each increasing level of boron significantly enhanced grain and stover yield in comparison to preceding lower level of boron. Application of $0.90 \mathrm{mg} \mathrm{B} \mathrm{kg}^{-1}$ increased grain yield by $8.7 \%$ and $13.1 \%$ and stover yield by $9.9 \%$ and $11.4 \%$ during first and second year, respectively, over control. The improvement in biomass yield of maize may be attributed to the complementary role of boron in the reproduction and vegetative stage of plants. The present finding is in agreement with that of Kumar et al., (2019).

\section{Nutrient content}

Lime had a significant positive effect on the nutrient content in both grain and stover of maize (Table 2). Irrespective of treatments and year, $\mathrm{N}$ content ranged from 0.98 to 1.21 $\%$ in grain and 0.55 to $0.69 \%$ in stover, $\mathrm{P}$ 
content from 0.37 to $0.46 \%$ in grain and 0.12 to $0.17 \%$ in stover, $\mathrm{K}$ content from 0.52 to $0.63 \%$ in grain and 1.08 to $1.17 \%$ in stover, $\mathrm{Ca}$ content from 0.34 to $0.56 \%$ in grain and 0.35 to $0.57 \%$ in stover and B content from 9.01 to $12.60 \mathrm{mg} \mathrm{kg}^{-1}$ in grain and 3.16 to $4.21 \mathrm{mg} \mathrm{kg}^{-1}$ in stover. Increase in nutrient content in plants with lime application might be due to release of plant nutrients in available form into soil solution as a result of neutralization of soil acidity. Effect of phosphorus on $\mathrm{N}, \mathrm{P}, \mathrm{K}$ and $\mathrm{Ca}$ content in maize was found to be significant, while impact on B content was insignificant. Maximum values of $\mathrm{N}, \mathrm{P}$ and $\mathrm{K}$ content in grain and $\mathrm{N}$ and $\mathrm{K}$ content in stover was observed with $40.2 \mathrm{mg} \mathrm{P}_{2} \mathrm{O}_{5} \mathrm{~kg}^{-1}$ which was at par with $26.8 \mathrm{mg} \mathrm{P}_{2} \mathrm{O}_{5} \mathrm{~kg}^{-1}$ during first and second year. On the other hand, maximum $\mathrm{P}$ content in stover was recorded at $26.8 \mathrm{mg}$
$\mathrm{P}_{2} \mathrm{O}_{5} \mathrm{~kg}^{-1}$ which was at par with $13.4 \mathrm{mg} \mathrm{P}_{2} \mathrm{O}_{5}$ $\mathrm{kg}^{-1}$ during both the years. Highest $\mathrm{Ca}$ content was observed at $40.2 \mathrm{mg} \mathrm{P}_{2} \mathrm{O}_{5} \mathrm{~kg}^{-1}$ which was found to be at par with the application of 13.4 $\mathrm{mg} \mathrm{P}_{2} \mathrm{O}_{5} \mathrm{~kg}^{-1}$ and $26.8 \mathrm{mg} \mathrm{P}_{2} \mathrm{O}_{5} \mathrm{~kg}^{-1}$ in both grain and stover during first as well as second year. Increase in nutrient absorption due to phosphorus application might be due to its positive effect on better root system (Sharma et al., 2008) which helps the plant to effectively absorption of nutrients from the soil. Effect of boron was significant on N, P and $\mathrm{B}$ content in grain and stover. Highest $\mathrm{N}$, $\mathrm{P}$ and $\mathrm{B}$ content in both grain and stover was observed at $0.90 \mathrm{mg} \mathrm{B} \mathrm{kg}^{-1}$ which was at par with $0.45 \mathrm{mg} \mathrm{B} \mathrm{kg}^{-1}$ during both the years. Positive effect of boron on N, P and B contents in maize have been reported by Adem et al., (2011); Barman et al., (2014).

Table.1 Effect of lime, phosphorus and boron on grain and stover yield of maize

\begin{tabular}{|c|c|c|c|c|}
\hline \multirow[t]{2}{*}{ Treatments } & \multicolumn{2}{|c|}{ Grain yield $\left(\mathrm{g} \mathrm{pot}^{-1}\right)$} & \multicolumn{2}{|c|}{ Stover yield $\left(\mathrm{g} \mathrm{pot}^{-1}\right)$} \\
\hline & I year & II year & I year & II year \\
\hline \multicolumn{5}{|l|}{ Lime levels } \\
\hline $\mathbf{0}$ & 61.93 & 62.43 & 101.66 & 102.47 \\
\hline $1 / 4$ lime of LR & 77.63 & 77.31 & 126.21 & 124.93 \\
\hline SEm \pm & 0.26 & 0.38 & 1.00 & 0.80 \\
\hline $\mathrm{CD}(\mathrm{P}=0.05)$ & 0.73 & 1.08 & 2.83 & 2.28 \\
\hline \multicolumn{5}{|c|}{ Phosphorus levels (mg kg ${ }^{-1}$ ) } \\
\hline $\mathbf{0}$ & 59.70 & 60.15 & 101.46 & 100.56 \\
\hline 13.4 & 70.97 & 71.09 & 110.37 & 109.30 \\
\hline 26.8 & 73.78 & 73.92 & 121.45 & 120.64 \\
\hline 40.2 & 74.66 & 74.30 & 122.46 & 124.28 \\
\hline SEm \pm & 0.36 & 0.54 & 1.41 & 1.13 \\
\hline $\mathrm{CD}(\mathrm{P}=0.05)$ & 1.03 & 1.53 & 4.01 & 3.22 \\
\hline \multicolumn{5}{|c|}{ Boron levels ( $\mathrm{mg} \mathrm{kg}^{-1}$ ) } \\
\hline $\mathbf{0}$ & 66.19 & 64.79 & 108.57 & 105.85 \\
\hline 0.45 & 71.19 & 71.52 & 113.88 & 117.36 \\
\hline 0.90 & 71.96 & 73.30 & 119.35 & 117.88 \\
\hline SEm \pm & 0.31 & 0.47 & 1.22 & 0.98 \\
\hline $\mathrm{CD}(\mathrm{P}=\mathbf{0 . 0 5})$ & 0.89 & 1.33 & 3.47 & 2.79 \\
\hline
\end{tabular}


Table.2 Effect of lime, phosphorus and boron on nitrogen, phosphorus, potassium, calcium and boron content of maize

\begin{tabular}{|c|c|c|c|c|c|c|c|c|c|c|c|c|c|c|c|c|c|c|c|c|}
\hline \multirow[t]{3}{*}{ Treatments } & \multicolumn{4}{|c|}{ Nitrogen content (\%) } & \multicolumn{4}{|c|}{ Phosphorus content (\%) } & \multicolumn{4}{|c|}{ Potassium content (\%) } & \multicolumn{4}{|c|}{ Calcium content (\%) } & \multicolumn{4}{|c|}{ Boron content (mg kg ${ }^{-1}$ ) } \\
\hline & \multicolumn{2}{|c|}{ Grain } & \multicolumn{2}{|c|}{ Stover } & \multicolumn{2}{|c|}{ Grain } & \multicolumn{2}{|c|}{ Stover } & \multicolumn{2}{|c|}{ Grain } & \multicolumn{2}{|c|}{ Stover } & \multicolumn{2}{|c|}{ Grain } & \multicolumn{2}{|c|}{ Stover } & \multicolumn{2}{|c|}{ Grain } & \multicolumn{2}{|c|}{ Stover } \\
\hline & I year & II year & I year & II year & I year & II year & I year & II year & $\begin{array}{c}\text { I } \\
\text { year }\end{array}$ & $\begin{array}{c}\text { II } \\
\text { year }\end{array}$ & $\begin{array}{c}\text { I } \\
\text { year }\end{array}$ & $\begin{array}{c}\text { II } \\
\text { year }\end{array}$ & $\begin{array}{c}\text { I } \\
\text { year }\end{array}$ & $\begin{array}{c}\text { II } \\
\text { year }\end{array}$ & $\begin{array}{c}\text { I } \\
\text { year }\end{array}$ & $\begin{array}{c}\text { II } \\
\text { year }\end{array}$ & I year & II year & I year & $\begin{array}{c}\text { II } \\
\text { year }\end{array}$ \\
\hline \multicolumn{21}{|l|}{ Lime levels } \\
\hline $\mathbf{0}$ & 0.98 & 1.01 & 0.55 & 0.57 & 0.37 & 0.39 & 0.12 & 0.15 & 0.53 & 0.57 & 1.10 & 1.08 & 0.35 & 0.34 & 0.36 & 0.35 & 10.81 & 10.86 & 3.73 & 3.76 \\
\hline $1 / 4$ lime of LR & 1.19 & 1.21 & 0.69 & 0.68 & 0.43 & 0.46 & 0.15 & 0.16 & 0.59 & 0.62 & 1.15 & 1.14 & 0.56 & 0.55 & 0.57 & 0.57 & 11.03 & 11.08 & 3.93 & 3.95 \\
\hline SEm \pm & 0.011 & 0.010 & 0.014 & 0.013 & 0.008 & 0.009 & 0.007 & 0.005 & $\begin{array}{c}0.01 \\
2\end{array}$ & $\begin{array}{c}0.01 \\
2\end{array}$ & $\begin{array}{c}0.01 \\
0\end{array}$ & $\begin{array}{c}0.00 \\
9\end{array}$ & $\begin{array}{c}0.01 \\
9\end{array}$ & $\begin{array}{c}0.01 \\
9\end{array}$ & $\begin{array}{c}0.01 \\
2\end{array}$ & $\begin{array}{c}0.01 \\
3\end{array}$ & 0.008 & 0.008 & 0.010 & 0.009 \\
\hline $\mathrm{CD}(\mathrm{P}=\mathbf{0 . 0 5})$ & 0.032 & 0.029 & 0.039 & 0.037 & 0.023 & 0.025 & 0.021 & 0.015 & $\begin{array}{c}0.03 \\
3\end{array}$ & $\begin{array}{c}0.03 \\
5\end{array}$ & $\begin{array}{c}0.02 \\
7\end{array}$ & $\begin{array}{c}0.02 \\
6\end{array}$ & $\begin{array}{c}0.05 \\
3\end{array}$ & $\begin{array}{c}0.05 \\
4\end{array}$ & $\begin{array}{c}0.03 \\
4\end{array}$ & $\begin{array}{c}0.03 \\
7\end{array}$ & 0.022 & 0.022 & 0.029 & 0.025 \\
\hline \multicolumn{21}{|c|}{ Phosphorus levels (mg kg ${ }^{-1}$ ) } \\
\hline $\mathbf{0}$ & 1.02 & 1.04 & 0.58 & 0.56 & 0.34 & 0.36 & 0.11 & 0.14 & 0.52 & 0.54 & 1.08 & 1.05 & 0.38 & 0.38 & 0.40 & 0.41 & 10.91 & 10.97 & 3.82 & 3.84 \\
\hline 13.4 & 1.10 & 1.11 & 0.61 & 0.59 & 0.40 & 0.44 & 0.13 & 0.16 & 0.55 & 0.60 & 1.10 & 1.09 & 0.47 & 0.44 & 0.47 & 0.48 & 10.93 & 10.96 & 3.83 & 3.85 \\
\hline 26.8 & 1.12 & 1.13 & 0.63 & 0.66 & 0.43 & 0.45 & 0.15 & 0.17 & 0.58 & 0.60 & 1.15 & 1.13 & 0.49 & 0.48 & 0.46 & 0.48 & 10.92 & 10.98 & 3.85 & 3.87 \\
\hline 40.2 & 1.11 & 1.17 & 0.66 & 0.68 & 0.44 & 0.45 & 0.15 & 0.16 & 0.59 & 0.63 & 1.17 & 1.16 & 0.49 & 0.49 & 0.52 & 0.49 & 10.91 & 10.96 & 3.84 & 3.87 \\
\hline SEm \pm & 0.016 & 0.015 & 0.019 & 0.018 & 0.011 & 0.013 & 0.010 & 0.008 & $\begin{array}{c}0.01 \\
7\end{array}$ & $\begin{array}{c}0.01 \\
7\end{array}$ & $\begin{array}{c}0.01 \\
4\end{array}$ & $\begin{array}{c}0.01 \\
3\end{array}$ & $\begin{array}{c}0.02 \\
6\end{array}$ & $\begin{array}{c}0.02 \\
7\end{array}$ & $\begin{array}{c}0.01 \\
7\end{array}$ & $\begin{array}{c}0.01 \\
8\end{array}$ & 0.011 & 0.011 & 0.014 & 0.013 \\
\hline$C D(P=0.05)$ & 0.045 & 0.041 & 0.055 & 0.052 & 0.032 & 0.036 & 0.029 & 0.022 & $\begin{array}{c}0.04 \\
7\end{array}$ & $\begin{array}{c}0.04 \\
9\end{array}$ & $\begin{array}{c}0.03 \\
9\end{array}$ & $\begin{array}{c}0.03 \\
6\end{array}$ & $\begin{array}{c}0.07 \\
5\end{array}$ & $\begin{array}{c}0.07 \\
6\end{array}$ & $\begin{array}{c}0.04 \\
9\end{array}$ & $\begin{array}{c}0.05 \\
2\end{array}$ & NS & NS & NS & NS \\
\hline \multicolumn{21}{|c|}{ Boron levels (mg kg ${ }^{-1}$ ) } \\
\hline $\mathbf{0}$ & 1.02 & 1.05 & 0.58 & 0.58 & 0.38 & 0.39 & 0.12 & 0.13 & 0.55 & 0.59 & 1.12 & 1.10 & 0.47 & 0.46 & 0.47 & 0.47 & 9.01 & 9.07 & 3.16 & 3.20 \\
\hline 0.45 & 1.11 & 1.13 & 0.62 & 0.62 & 0.40 & 0.44 & 0.13 & 0.17 & 0.57 & 0.60 & 1.13 & 1.11 & 0.46 & 0.44 & 0.47 & 0.47 & 11.20 & 11.25 & 4.16 & 4.18 \\
\hline 0.90 & 1.12 & 1.14 & 0.66 & 0.67 & 0.43 & 0.45 & 0.15 & 0.17 & 0.56 & 0.59 & 1.13 & 1.10 & 0.45 & 0.44 & 0.46 & 0.46 & 12.55 & 12.60 & 4.18 & 4.21 \\
\hline SEm \pm & 0.014 & 0.013 & 0.017 & 0.016 & 0.010 & 0.011 & 0.009 & 0.007 & $\begin{array}{c}0.01 \\
4\end{array}$ & $\begin{array}{c}0.01 \\
5\end{array}$ & $\begin{array}{c}0.01 \\
2\end{array}$ & $\begin{array}{c}0.01 \\
1\end{array}$ & $\begin{array}{c}0.02 \\
3\end{array}$ & $\begin{array}{c}0.02 \\
3\end{array}$ & $\begin{array}{c}0.01 \\
5\end{array}$ & $\begin{array}{c}0.01 \\
6\end{array}$ & 0.010 & 0.010 & 0.012 & 0.011 \\
\hline $\mathrm{CD}(\mathrm{P}=\mathbf{0 . 0 5})$ & 0.039 & 0.036 & 0.048 & 0.045 & 0.028 & 0.031 & 0.025 & 0.019 & NS & NS & NS & NS & NS & NS & NS & NS & 0.027 & 0.027 & 0.035 & 0.031 \\
\hline
\end{tabular}


Table.3 Effect of lime, phosphorus and boron on nitrogen, phosphorus, potassium, calcium and boron uptake of maize

\begin{tabular}{|c|c|c|c|c|c|c|c|c|c|c|c|c|c|c|c|c|c|c|c|c|}
\hline \multirow[t]{3}{*}{ Treatments } & \multicolumn{4}{|c|}{ N uptake (mg pot $\left.{ }^{-1}\right)$} & \multicolumn{4}{|c|}{ P uptake (mg pot $\left.{ }^{-1}\right)$} & \multicolumn{4}{|c|}{ K uptake (mg pot $\left.{ }^{-1}\right)$} & \multicolumn{4}{|c|}{ Ca uptake $\left(\mathrm{mg} \mathrm{pot}^{-1}\right)$} & \multicolumn{4}{|c|}{ B uptake $\left(\mu \mathrm{g} \operatorname{pot}^{-1}\right)$} \\
\hline & \multicolumn{2}{|c|}{ Grain } & \multicolumn{2}{|c|}{ Stover } & \multicolumn{2}{|c|}{ Grain } & \multicolumn{2}{|c|}{ Stover } & \multicolumn{2}{|c|}{ Grain } & \multicolumn{2}{|c|}{ Stover } & \multicolumn{2}{|c|}{ Grain } & \multicolumn{2}{|c|}{ Stover } & \multicolumn{2}{|c|}{ Grain } & \multicolumn{2}{|c|}{ Stover } \\
\hline & $\begin{array}{c}\text { I } \\
\text { year }\end{array}$ & $\begin{array}{c}\text { II } \\
\text { year }\end{array}$ & $\begin{array}{c}\text { I } \\
\text { year }\end{array}$ & $\begin{array}{c}\text { II } \\
\text { year }\end{array}$ & $\begin{array}{c}\mathrm{I} \\
\text { year }\end{array}$ & $\begin{array}{c}\text { II } \\
\text { year }\end{array}$ & $\begin{array}{c}\text { I } \\
\text { year }\end{array}$ & $\begin{array}{c}\text { II } \\
\text { year }\end{array}$ & $\begin{array}{c}\text { I } \\
\text { year }\end{array}$ & $\begin{array}{c}\text { II } \\
\text { year }\end{array}$ & I year & II year & I year & $\begin{array}{c}\text { II } \\
\text { year }\end{array}$ & I year & $\begin{array}{c}\text { II } \\
\text { year }\end{array}$ & I year & $\begin{array}{c}\text { II } \\
\text { year }\end{array}$ & I year & $\begin{array}{c}\text { II } \\
\text { year }\end{array}$ \\
\hline \multicolumn{21}{|l|}{ Lime levels } \\
\hline $\mathbf{0}$ & 611.6 & 634.1 & 561.7 & 586.4 & 230.1 & 247.8 & 123.2 & 152.7 & 329.2 & 355.4 & 1120.7 & 1105.6 & 220.7 & 216.1 & 366.8 & 364.4 & 672.9 & 682.8 & 381.3 & 388.3 \\
\hline $1 / 4$ lime of $L R$ & 927.1 & 939.5 & 872.3 & 853.8 & 338.5 & 358.9 & 190.5 & 208.0 & 459.0 & 483.7 & 1459.0 & 1423.8 & 440.2 & 427.3 & 723.5 & 719.8 & 859.8 & 862.0 & 498.5 & 496.7 \\
\hline SEm \pm & 7.2 & 7.3 & 15.3 & 14.9 & 5.3 & 5.9 & 8.1 & 6.0 & 7.3 & 8.3 & 15.5 & 13.8 & 13.2 & 13.6 & 14.9 & 13.9 & 2.8 & 4.0 & 4.0 & 3.1 \\
\hline $\mathrm{CD}(\mathbf{P}=\mathbf{0 . 0 5})$ & 20.6 & 20.7 & 43.5 & 42.4 & 15.0 & 16.7 & 23.2 & 16.9 & 20.9 & 23.5 & 43.9 & 39.2 & 37.4 & 38.7 & 42.2 & 39.4 & 8.1 & 11.3 & 11.4 & 8.8 \\
\hline \multicolumn{21}{|c|}{ Phosphorus levels (mg kg-1) } \\
\hline $\mathbf{0}$ & 614.9 & 633.7 & 591.8 & 569.6 & 204.7 & 218.8 & 111.3 & 137.5 & 312.6 & 329.7 & 1100.6 & 1058.1 & 232.1 & 231.9 & 419.4 & 424.0 & 656.4 & 666.2 & 390.0 & 390.1 \\
\hline 13.4 & 787.2 & 795.0 & 679.6 & 657.5 & 283.9 & 316.4 & 150.1 & 177.4 & 389.8 & 429.0 & 1219.8 & 1195.1 & 345.7 & 322.5 & 532.5 & 534.5 & 779.7 & 785.8 & 425.8 & 424.3 \\
\hline 26.8 & 839.7 & 842.0 & 775.9 & 797.1 & 317.8 & 337.4 & 181.2 & 206.2 & 430.1 & 446.9 & 1404.6 & 1366.1 & 369.5 & 360.6 & 579.4 & 591.2 & 810.6 & 817.6 & 470.8 & 470.9 \\
\hline 40.2 & 835.5 & 876.6 & 820.7 & 856.1 & 330.8 & 340.6 & 184.9 & 200.4 & 444.1 & 472.5 & 1434.7 & 1439.6 & 374.6 & 371.6 & 649.3 & 618.9 & 818.8 & 820.1 & 473.0 & 484.5 \\
\hline SEm \pm & 10.2 & 10.3 & 21.7 & 21.1 & 7.5 & 8.3 & 11.5 & 8.4 & 10.4 & 11.7 & 21.9 & 19.5 & 18.6 & 19.3 & 21.0 & 19.6 & 4.0 & 5.6 & 5.7 & 4.4 \\
\hline $\mathrm{CD}(\mathrm{P}=\mathbf{0 . 0 5})$ & 29.1 & 29.3 & 61.6 & 60.0 & 21.3 & 23.6 & 32.8 & 23.9 & 29.5 & 33.2 & 62.1 & 55.4 & 52.9 & 54.7 & 59.7 & 55.7 & 11.4 & 16.0 & 16.1 & 12.5 \\
\hline \multicolumn{21}{|c|}{ Boron levels ( $\mathbf{m ~ k g}^{-1}$ ) } \\
\hline $\mathbf{0}$ & 687.9 & 691.3 & 637.4 & 619.3 & 253.3 & 256.3 & 134.0 & 140.5 & 367.5 & 387.0 & 1221.2 & 1172.8 & 324.0 & 306.0 & 523.5 & 509.5 & 597.1 & 588.1 & 344.6 & 339.0 \\
\hline 0.45 & 803.4 & 821.2 & 718.4 & 743.1 & 287.2 & 318.4 & 149.5 & 199.8 & 407.0 & 432.7 & 1293.7 & 1312.8 & 335.8 & 324.0 & 551.5 & 562.3 & 798.0 & 805.1 & 474.8 & 491.3 \\
\hline 0.90 & 816.7 & 847.9 & 795.3 & 797.9 & 312.4 & 335.2 & 187.1 & 200.9 & 407.9 & 439.0 & 1354.8 & 1308.5 & 331.5 & 335.0 & 560.4 & 554.6 & 904.0 & 924.1 & 500.3 & 497.1 \\
\hline SEm \pm & 8.9 & 8.9 & 18.8 & 18.3 & 6.5 & 7.2 & 10.0 & 7.3 & 9.0 & 10.1 & 18.9 & 16.9 & 16.1 & 16.7 & 18.2 & 17.0 & 3.5 & 4.9 & 4.9 & 3.8 \\
\hline $\mathrm{CD}(\mathbf{P}=\mathbf{0 . 0 5})$ & 25.2 & 25.4 & 53.3 & 51.9 & 18.4 & 20.4 & 28.4 & 20.7 & 25.6 & 28.8 & 53.8 & 48.0 & NS & NS & NS & NS & 9.9 & 13.9 & 14.0 & 10.8 \\
\hline
\end{tabular}


Table.4 Effect of lime, phosphorus and boron on soil chemical properties of post-harvest soil

\begin{tabular}{|c|c|c|c|c|c|c|c|c|c|c|c|c|c|c|c|c|c|c|c|c|c|c|}
\hline \multirow[t]{2}{*}{ Treatments } & \multicolumn{2}{|c|}{ pH } & \multicolumn{2}{|c|}{$\begin{array}{l}\text { Organic } \\
\text { carbon } \\
\left(\mathrm{g} \mathrm{kg}^{-1}\right)\end{array}$} & \multicolumn{2}{|c|}{$\begin{array}{c}\text { Base } \\
\text { saturation } \\
(\%)\end{array}$} & \multicolumn{2}{|c|}{$\begin{array}{c}\text { Available N } \\
\left(\mathrm{kg} \mathrm{ha}^{-1}\right)\end{array}$} & \multicolumn{2}{|c|}{$\begin{array}{c}\text { Available P } \\
\left(\mathrm{kg} \mathrm{ha}^{-1}\right)\end{array}$} & \multicolumn{2}{|c|}{$\begin{array}{c}\text { Available K } \\
\left(\mathrm{kg} \mathrm{ha}^{-1}\right)\end{array}$} & \multicolumn{2}{|c|}{$\begin{array}{l}\text { Exch. Ca } \\
{\left[\text { cmolkg }^{-1}\right]}\end{array}$} & \multicolumn{2}{|c|}{$\begin{array}{c}\text { Available B } \\
\left(\mathrm{mg} \mathrm{kg}^{-1}\right)\end{array}$} & \multicolumn{2}{|c|}{$\begin{array}{c}\text { Exch. } \mathbf{H}^{+} \\
\left(\mathbf{c m o l} \mathbf{k g}^{-1}\right)\end{array}$} & \multicolumn{2}{|c|}{$\begin{array}{l}\text { Exch. } \mathbf{A l}^{3+} \\
\left(\mathbf{c m o l} \mathbf{k g}^{-1}\right)\end{array}$} & \multicolumn{2}{|c|}{$\begin{array}{c}\text { Total } \\
\text { potential } \\
\text { acidity } \\
\left(\mathbf{c m o l} \mathbf{k g}^{-1}\right)\end{array}$} \\
\hline & $\begin{array}{l}\text { I } \\
\text { year }\end{array}$ & $\begin{array}{l}\text { II } \\
\text { year }\end{array}$ & $\begin{array}{l}\text { I } \\
\text { year }\end{array}$ & $\begin{array}{l}\text { II } \\
\text { year }\end{array}$ & $\begin{array}{l}\text { I } \\
\text { year }\end{array}$ & $\begin{array}{l}\text { II } \\
\text { year }\end{array}$ & $\begin{array}{l}\text { I } \\
\text { year }\end{array}$ & $\begin{array}{l}\text { II } \\
\text { year }\end{array}$ & $\begin{array}{l}\text { I } \\
\text { year }\end{array}$ & $\begin{array}{l}\text { II } \\
\text { year }\end{array}$ & $\begin{array}{l}\text { I } \\
\text { year }\end{array}$ & $\begin{array}{l}\text { II } \\
\text { year }\end{array}$ & $\begin{array}{l}\text { I } \\
\text { year }\end{array}$ & $\begin{array}{l}\text { II } \\
\text { year }\end{array}$ & $\begin{array}{l}\text { I } \\
\text { year }\end{array}$ & $\begin{array}{l}\text { II } \\
\text { year }\end{array}$ & $\begin{array}{l}\text { I } \\
\text { year }\end{array}$ & $\begin{array}{l}\text { II } \\
\text { year }\end{array}$ & $\begin{array}{l}\text { I } \\
\text { year }\end{array}$ & $\begin{array}{l}\text { II } \\
\text { year }\end{array}$ & $\begin{array}{l}\text { I } \\
\text { year }\end{array}$ & $\begin{array}{l}\text { II } \\
\text { year }\end{array}$ \\
\hline \multicolumn{23}{|l|}{ Lime levels } \\
\hline 0 & 5.22 & 5.20 & 15.6 & 15.8 & 26.0 & 26.7 & 238.6 & 240.0 & 10.6 & 10.4 & 149.9 & 150.0 & 3.04 & 3.06 & 0.51 & 0.54 & 0.99 & 0.92 & 1.32 & 1.34 & 14.90 & 14.83 \\
\hline $1 / 4$ lime of LR & 5.65 & 5.69 & 15.7 & 15.9 & 31.0 & 31.6 & 248.7 & 249.0 & 12.2 & 12.5 & 151.0 & 151.6 & 4.23 & 4.27 & 0.56 & 0.58 & 0.55 & 0.64 & 1.22 & 1.21 & 13.20 & 13.17 \\
\hline SEm \pm & 0.02 & 0.02 & 0.02 & 0.02 & 0.30 & 0.30 & 0.31 & 0.24 & 0.15 & 0.19 & 0.24 & 0.28 & 0.015 & 0.013 & 0.015 & 0.013 & 0.01 & 0.01 & 0.01 & 0.01 & 0.02 & 0.01 \\
\hline CD (P=0.05) & 0.07 & 0.06 & NS & NS & 0.84 & 0.86 & 0.88 & 0.68 & 0.41 & 0.54 & 0.68 & 0.78 & 0.042 & 0.037 & 0.044 & 0.038 & 0.03 & 0.02 & 0.03 & 0.02 & 0.05 & 0.03 \\
\hline \multicolumn{23}{|c|}{ Phosphorus levels $\left(\mathrm{mg} \mathrm{kg}^{-1}\right)$} \\
\hline $\mathbf{0}$ & 5.40 & 5.41 & 15.5 & 15.7 & 28.6 & 29.3 & 243.0 & 244.0 & 10.1 & 10.0 & 150.7 & 150.7 & 2.89 & 2.93 & 0.54 & 0.55 & 0.78 & 0.79 & 1.27 & 1.27 & 14.07 & 14.02 \\
\hline 13.4 & 5.49 & 5.48 & 15.7 & 15.9 & 28.7 & 29.3 & 243.5 & 244.4 & 11.2 & 11.2 & 150.4 & 150.6 & 3.80 & 3.83 & 0.52 & 0.57 & 0.75 & 0.76 & 1.28 & 1.29 & 14.04 & 14.00 \\
\hline 26.8 & 5.44 & 5.45 & 15.8 & 16.0 & 28.5 & 29.1 & 244.1 & 244.5 & 12.1 & 12.2 & 150.3 & 150.6 & 3.92 & 3.94 & 0.53 & 0.56 & 0.78 & 0.79 & 1.26 & 1.28 & 14.06 & 13.99 \\
\hline 40.2 & 5.41 & 5.43 & 1.57 & 15.9 & 28.2 & 29.0 & 244.2 & 245.0 & 12.3 & 12.4 & 150.2 & 151.3 & 3.92 & 3.95 & 0.54 & 0.57 & 0.76 & 0.78 & 1.27 & 1.28 & 14.04 & 13.98 \\
\hline SEm \pm & 0.03 & 0.03 & 0.02 & 0.02 & 0.42 & 0.43 & 0.44 & 0.34 & 0.21 & 0.27 & 0.34 & 0.39 & 0.021 & 0.018 & 0.022 & 0.019 & 0.02 & 0.01 & 0.01 & 0.01 & 0.02 & 0.02 \\
\hline $\mathrm{CD}(\mathbf{P}=0.05)$ & NS & NS & NS & NS & NS & NS & NS & NS & 0.58 & 0.77 & NS & NS & 0.060 & 0.052 & NS & NS & NS & NS & NS & NS & NS & NS \\
\hline \multicolumn{23}{|c|}{ Boron levels $\left(\mathrm{mg} \mathrm{kg}^{-1}\right)$} \\
\hline 0 & 5.44 & 5.44 & 15.5 & 15.8 & 28.5 & 29.1 & 243.3 & 244.0 & 11.4 & 11.3 & 150.5 & 150.8 & 3.64 & 3.66 & 0.47 & 0.52 & 0.77 & 0.78 & 1.26 & 1.27 & 14.04 & 13.99 \\
\hline 0.45 & 5.43 & 5.44 & 15.7 & 15.8 & 28.5 & 29.2 & 244.0 & 244.7 & 11.4 & 11.5 & 150.2 & 150.5 & 3.64 & 3.67 & 0.54 & 0.56 & 0.77 & 0.78 & 1.27 & 1.28 & 14.05 & 14.00 \\
\hline 0.90 & 5.44 & 5.45 & 15.8 & 15.9 & 28.5 & 29.1 & 243.8 & 244.7 & 11.5 & 11.6 & 150.6 & 151.0 & 3.63 & 3.66 & 0.59 & 0.61 & 0.77 & 0.77 & 1.28 & 1.29 & 14.07 & 14.01 \\
\hline SEm \pm & 0.03 & 0.03 & 0.02 & 0.02 & 0.36 & 0.37 & 0.38 & 0.29 & 0.18 & 0.23 & 0.29 & 0.34 & 0.018 & 0.016 & 0.019 & 0.016 & 0.01 & 0.01 & 0.01 & 0.01 & 0.02 & 0.01 \\
\hline $\mathrm{CD}(\mathbf{P}=\mathbf{0 . 0 5})$ & NS & NS & NS & NS & NS & NS & NS & NS & NS & NS & NS & NS & NS & NS & 0.054 & 0.047 & NS & NS & NS & NS & NS & NS \\
\hline
\end{tabular}




\section{Nutrient uptake}

Effect of lime on nutrient uptake in grain and stover showed positive significant response (Table 3). It is apparent from the data that maximum nutrient uptake was recorded with $1 / 4$ lime of LR for grain and stover during first as well as second year. Application of $1 / 4$ lime of LR increased the mean N, P, K, Ca and B uptake in grain by 49.8, 45.9, 37.7, 98.6 and $27.0 \%$ and in stover by $50.3,44.4,29.5,97.4$ and $29.3 \%$, respectively over control. Lime application enhanced grain and stover yield as well as nutrient concentration which in resulted increased nutrient uptake by crop (Yadesa et al., 2019). Effect of phosphorus on nutrient uptake was significant where maximum uptake values were obtained with application of $40.2 \mathrm{mg} \mathrm{P}_{2} \mathrm{O}_{5} \mathrm{~kg}^{-1}$. But these uptake values were at par with $26.8 \mathrm{mg} \mathrm{kg}^{-1}$ $\mathrm{P}_{2} \mathrm{O}_{5}$ during both the years. Application of $26.8 \mathrm{mg} \mathrm{kg}^{-1} \mathrm{P}_{2} \mathrm{O}_{5}$ enhanced mean $\mathrm{N}, \mathrm{P}, \mathrm{K}, \mathrm{Ca}$ and $\mathrm{B}$ uptake in grain by $34.7,54.7,36.5$, 57.4 and $23.1 \%$ and in stover by $35.4,55.7$, $28.4,38.8$ and $20.7 \%$, respectively over control. Increase in uptake of nutrients due to phosphorus application may be explained on the ground that the addition of phosphorus resulted in more absorption of nutrients by plants due to improvement in root growth through increased root hair length, besides increasing the root surface area (Darwesh et al., 2013).

Boron application significantly enhanced $\mathrm{N}$, $\mathrm{P}, \mathrm{K}$ and $\mathrm{B}$ uptake in grain and stover, while $\mathrm{Ca}$ uptake was unaffected. Maximum uptake values in grain as well as stover was recorded with $0.90 \mathrm{mg} \mathrm{B} \mathrm{kg}{ }^{-1}$ during both the years. Application of $0.90 \mathrm{mg} \mathrm{B} \mathrm{kg}^{-1}$ increased the mean $\mathrm{N}, \mathrm{P}, \mathrm{K}$ and B uptake in grain by 20.7 , $27.1,12.2$ and $54.2 \%$ and in stover by 26.7, $41.3,11.2$ and $45.9 \%$, respectively over control. Affirmative effect of boron on nutrient uptake was reported by Barman et al., (2014); Sahin (2014).

\section{Soil properties}

It is apparent from the data that lime had a significant positive effect on soil $\mathrm{pH}$ and base saturation, where mean $\mathrm{pH}$ range increased from $\mathrm{pH} 5.21$ to 5.67 and mean base saturation from 26.3 to $31.3 \%$ (Table 4 ). The increase in soil $\mathrm{pH}$ and percent base saturation due to liming may be due to displacement and replacement of acidic cations like $\mathrm{Al}^{3+}, \mathrm{H}^{+}$ and $\mathrm{Fe}^{3+}$ ions by $\mathrm{Ca}^{2+}$ ions present in the liming material (Kisinyo et al., 2013). Application of lime also significantly improved the nutrient status of post-harvest soil. Available N, P, K, B and exchangeable $\mathrm{Ca}$ increased significantly with application of $1 / 4$ lime of LR during both the years, where mean available $\mathrm{N}, \mathrm{P}, \mathrm{K}, \mathrm{B}$ and mean exchangeable $\mathrm{Ca}$ increased from 239.3 to $248.9 \mathrm{~kg} \mathrm{ha}^{-1}, 10.5$ to $12.3 \mathrm{~kg} \mathrm{ha}^{-1}, 150.0$ to $151.3 \mathrm{~kg} \mathrm{ha}^{-1}, 0.52$ to $0.57 \mathrm{mg} \mathrm{kg}^{-1}$ and 3.05 to $4.25 \mathrm{cmol} \mathrm{kg}^{-1}$, respectively. Availability of these nutrients was more in the lime treated soil because liming has been shown to enhance the mineralization of organic matter, thereby releasing inorganic nutrients. Also, liming supplies $\mathrm{Ca}$ to the soil which freely occupies the exchange complex and hence its availability increases. Similar findings have been reported by Han et al., (2019). Increase in boron availability by liming may be due to neutralization of soil acidity which may have released boron into the soil solution (Sarkar $e t$ al., 2015). Effect of lime on soil acidity components was significant, where it significantly reduced exchangeable $\mathrm{H}^{+}, \mathrm{Al}^{3+}$ and total potential acidity of post-harvest soil during both the years. Application of $1 / 4$ lime of LR decreased the mean exchangeable $\mathrm{H}^{+}$, $\mathrm{Al}^{3+}$ and total potential acidity by $37.9 \%$, $8.3 \%$ and $11.3 \%$, respectively over control. A decrease in all forms of soil acidity due to liming has been reported by Badole et al., (2015). Organic carbon content of soil was not affected significantly with lime application. Application of phosphorus 
significantly improved the available $\mathrm{P}$ and exchangeable $\mathrm{Ca}$ of post-harvest soil where application of $40.2 \mathrm{mg} \mathrm{P}_{2} \mathrm{O}_{5} \mathrm{~kg}^{-1}$ showed maximum values. However, $40.2 \mathrm{mg} \mathrm{P}_{2} \mathrm{O}_{5} \mathrm{~kg}$ ${ }^{1}$ was found to be at par with $26.8 \mathrm{mg} \mathrm{P}_{2} \mathrm{O}_{5}$ $\mathrm{kg}^{-1}$ during both the years. At $26.8 \mathrm{mg} \mathrm{P}_{2} \mathrm{O}_{5}$ $\mathrm{kg}^{-1}$, mean available $\mathrm{P}$ increased from 10.0 to $12.2 \mathrm{~kg} \mathrm{ha}^{-1}$ and mean exchangeable Ca from 2.91 to $3.93 \mathrm{cmol} \mathrm{kg}^{-1}$. Increase in available $\mathrm{P}$ following phosphorus fertilization may be primarily due to the release of $\mathrm{P}$ from fertilizer. Similar results have also been reported by Venkatesh et al., (2002); Gadi et al., (2018).

Other soil properties did not show remarkable variation due to phosphorus application. Boron had a significant effect only on the available boron of post-harvest soil, while other soil properties remained unaffected. Application of $0.90 \mathrm{mg} \mathrm{B} \mathrm{kg}{ }^{-1}$ reflected maximum available boron, which increased the mean B content from 0.49 to $0.60 \mathrm{mg} \mathrm{kg}^{-1}$. The increase in available $\mathrm{B}$ with increasing rate of $\mathrm{B}$ application is in agreement with those of Barman et al., (2014).

From the above findings, it can be concluded that application of lime had an overall beneficial effect on the yield, nutrient content and uptake of maize as well as on the soil properties. Liming increased the soil $\mathrm{pH}$, base saturation, available $\mathrm{N}, \mathrm{P}, \mathrm{K}, \mathrm{Ca}$ and $\mathrm{B}$, while it decreased the exchangeable $\mathrm{H}^{+}, \mathrm{Al}^{3+}$ and total potential acidity of post-harvest soil. Phosphorus and boron application improved the yield, nutrient content and uptake of maize.

Improvement in soil $\mathrm{P}$ and $\mathrm{Ca}$ status due to $\mathrm{P}$ application and B status due to B application was also observed. Thus, application of 26.8 mg $\mathrm{P}_{2} \mathrm{O}_{5} \mathrm{~kg}^{-1}$ and $0.90 \mathrm{mg} \mathrm{B} \mathrm{kg}^{-1}$ along with liming at $1 / 4$ lime of LR may be recommended for better production of maize in acid soils of Nagaland.

\section{References}

Adem, G. N., Zametin, A., Asliham, E., Oguzhan, U., Sinan, A. and Metin, T. 2011. Yield and chemical composition of corn (Zea mays L.) as affected by boron management. International Journal of Plant, Animal and Environmental Science, 1(1): 42-51.

Agricultural Statistics at a Glance, 2019. Government of India, Ministry of Agriculture \& Farmers Welfare, Department of Ministry of Agriculture, Cooperation \& Farmers Welfare, Directorate of Economics and Statistics.

Badole, S., Datta, A., Basak, N., Seth, A., Padhan, D. and Mandal, B. 2015. Liming influences forms of acidity in soils belonging to different orders under Subtropical India. Communications in Soil Science and Plant Analysis, 46(16): 2079-2094.

Barman, M., Shukla, L. M., Datta, S. P. and Rattan, R. J. 2014. Effect of applied lime and boron on the availability of nutrients in an acid soil. Journal of Plant Nutrition, 37: 357-373.

Baruah, T. C. and Barthakur, H. P. 1997. A textbook of soil analysis. Vikas Publishing House Pvt. Ltd., 576 Masjid Road, Jangpura, New Delhi.

Bray, R. H. and Kurtz, L. T. 1945. Determination of total organic and available forms of phosphorus in soils. Soil Science, 59: 39-45.

Darwesh, D. A., Maulood, P. M. and Amin, S. A. 2013. effect of phosphorus fertilizers on growth and physiological phosphorus use efficiency of three soy bean cultivars. Journal of Agriculture and Veterinary Science, 3(6): 32-36.

Dible, W. T., Truog, E. and Berger, K. C. 1954. Boron determination in soils and plants: Simplified curcumin procedure. Analytical Chemistry, 26: 418-421. 
Fageria, N. K. and Baligar, V. C. 2008. Ameliorating soil acidity of tropical Oxisols by liming for sustainable crop production. In: Advances in Agronomy, volume 99 (ed. Sparks, D. L.), Academic Press. pp- 345-389.

Gadi, Y., Sharma, Y. K., Sharma, S. K. and Bordoloi, J. 2018. Influence of phosphorus and potassium on performance of green gram (Vigna radiata L.Wilczek) in Inceptisols of Nagaland. Annals of Plant and Soil Research, 20: 120-124.

Gaines, T. P. and Mitchell, G. A. 1979. Boron determination in soils and plants by the azomethine-H method. Communication in Soil Science and Plant Analysis, 10: 1099-1108.

Han, T., Cai, A., Liu, K., Huang, J., Wang, B., Li, D., Qaswar, M., Feng, G. and Zhang, H. 2019. The links between potassium availability and soil exchangeable calcium, magnesium, and aluminum are mediated by lime in acidic soil. Journal of Soils and Sediments, 19: 1382-1392.

Jackson, M. L. 1973. Soil Chemical Analysis. Prentice Hall of India Private Limited, New Delhi.

Kisinyo, P. O., Othieno, C. O., Gudu, S. O., Okalebo, J. R., Opala, P. A., Maghanga, J. K., Ng'etich,W. K., Agalo, J. J., Opile, R. W. and Kisinyo, J. A. 2013. Phosphorus sorption and lime requirements of maize growing acids soil of Kenya. Sustainable Agriculture Research, 2: 116-123.

Kumar, M., Singh, S., Singh, V., Singh, K. and Khanna, R. 2019. Effect of zinc and boron on growth and yield of maize (Zea mays L.). Progressive Research - An International Journal, 14(3): 215-221.

Kumar, S., Wani, J. A., Lone, B. A., Singh, P., Dar, Z. A., Qayoom, S. and Fayaz, A. 2017. Effect of different levels of phosphorus and sulphur on seed \& stover yield of soybean (Glycine max L. Merill) under 'Eutrochrepts'. Asian Research Journal of Agriculture, 5(1): 1-7.

Nazir, G., Sharma, U. and Kumar, P. 2016. Boron- its importance in crop production, status in Indian soils and crop responses to its application. International Journal of Advanced Research, 4(5): 654-660.

Ngosai, K. Sharma, Y.K., Sharma, S.K. and Bordoloi, J. 2018. Maize (Zea mays L) productivity and soil properties as affected by fertility levels under Nagaland condition. Annals of Plant and Soil Research, 20(2): 349-353.

Panse, V. G. and Sukhatme, P. V. 1961. Statistical methods for agricultural workers. Indian Council of Agricultural Research, New Delhi.

Sahin, S. 2014. Effect of boron fertilizer applications on the growth and $\mathrm{B}, \mathrm{N}$ uptake of maize (Zea mays L.) under the different soils. Journal of Food, Agriculture and Environment, 12(2): 1323-1327.

Sarkar, D., Ghosh, S., Batabyal, K., Mandal, B. and Chattopadhyay, A. P. 2015. Liming effects on extractable boron in six acidic soils. Communications in Soil Science and Plant Analysis, 46: 1320-1325.

Sarma, V.A.K., Krishnan, P., Budihal, S.L., 1987. Laboratory methods. NBSS pub.14, Technical Bulletin, National Bureau of Soil Survey and Land Use Planning, Nagpur, 49.

Sharma, R., Dahiya, S. S., Singh, M., Malik, R. K. and Singh, D. 2008. Effect of sulphur and phosphorus interactions on growth and nutrient content in green gram. Haryana Agricultural University Journal of Research, 38(1/2): 41-7.

Subbiah, B. V. and Asija, G. L. 1956. A rapid procedure for the determination of 
available nitrogen in soil. Current Science, 25: 259-60.

The, C., Calba, H., Zonkeng, C., Ngonkeu, E. L. M. and Adetimirin V. O. 2006. Response of maize grain yield to changes in acid soil characteristics after soil amendment. Plant and Soil, 284: 45-57.

Venkatesh, M. S., Majumdar, B., Kumar, K. and Patiram. 2002. Effect of phosphorus, FYM and lime on yield, $\mathrm{P}$ uptake by maize and forms of soil acidity in Typic Hapludalf of Meghalaya. Journal of the Indian Society of Soil Science, 50 (3): 254258.

Yadesa, W., Tadesse, A., Kibret, K. and Dechassa, N. 2019. Effect of liming and applied phosphorus on growth and $\mathrm{P}$ uptake of maize (Zea mays subsp.) plant grown in acid soils of West Wollega, Ethiopia. Journal of Plant Nutrition, 42(5): 477-490.

\section{How to cite this article:}

Merasenla Ao and Sharma, Y. K. 2020. Effect of Lime, Phosphorus and Boron on Yield and Chemical Composition of Maize (Zea mays L.) and Properties of Acid Soil of Nagaland. Int.J.Curr.Microbiol.App.Sci. 9(12): 398-408. doi: https://doi.org/10.20546/ijcmas.2020.912.050 